\title{
Rituximab therapy in autoimmune pulmonary alveolar proteinosis
}

\author{
R. Borie*, M-P. Debray", C. Laine ${ }^{\star}$, M. Aubier*, ${ }^{*+}$ and B. Crestani, ${ }^{*,+}$
}

\begin{abstract}
Idiopathic pulmonary alveolar proteinosis is presumed to be an autoimmune disorder that may lead to pulmonary insufficiency. However, steroids do not appear to be effective and the standard of therapy is whole-lung lavage. We report the first case of successful therapy with rituximab, which addresses the pathogenic mechanism of pulmonary alveolar proteinosis.
\end{abstract}

KEYWORDS: CD20, crazy paving, granulocyte-macrophage colony-stimulating factor, interstitial pneumonia, pulmonary alveolar proteinosis

$\mathbf{P}$ ulmonary alveolar proteinosis (PAP) is a rare disorder caused by accumulation of surfactant in pulmonary alveoli, which causes respiratory insufficiency [1-3]. For nearly $90 \%$ of cases, the disease is associated with neutralising autoantibodies against granulocyte-macrophage colony-stimulating factor (GM-CSF) [2].

The standard of care is symptomatic and consists of whole-lung lavage, which may result in numerous complications [4]. Other available therapies are based on GM-CSF supplementation, which is only effective in almost $50 \%$ of cases [5]. Rituximab is a monoclonal antibody directed against the CD20 antigen of B-lymphocytes. Since 1997, rituximab has been approved by the US Food and Drug Administration for the treatment of B-lymphoma. Moreover, rituximab has been demonstrated to be effective in various diseases mediated by autoantibodies, such as rheumatoid arthritis, idiopathic thrombocytopaenic purpura and systemic lupus erythematosus [6, 7]. We hypothesised that rituximab could provide clinical benefit for a patient with autoimmune PAP.

\section{CASE REPORT}

The diagnosis of autoimmune alveolar proteinosis was made in a 41-yr-old nonsmoker who presented with a dry cough without any remarkable finding on physical examination. The chest radiograph revealed bilateral infiltrates and highresolution computed tomography (HRCT) showed characteristic diffuse ground-glass opacification superimposed on a reticular pattern. The bronchoalveolar lavage was opaque and contained large and foamy alveolar macrophage and an increased number (43\%) of lymphocytes.
Bacterial, mycobacterial and fungal cultures were negative. The transbronchial lung biopsy showed near completed filling of the alveolar space and terminal bronchioles by periodic acid-Schiff positive acellular surfactant and confirmed the diagnosis of PAP. A careful review of the clinical history did not detect any exposure to inhaled toxics. HIV testing was negative. The serum antiGM-CSF immunoglobulin (Ig)G concentration was measured using a competition ELISA adapted from TANAKA et al. [8]. Anti-GM-CSF IgG was detected in the serum by ELISA (concentration: $319 \mu \mathrm{g} \cdot \mathrm{mL}^{-1}$ ). A diagnosis of idiopathic PAP was made. At the time of diagnosis, pulmonary function tests were within the normal range (diffusing capacity of the lung for carbon monoxide (DL,CO) $84 \%$ of the predicted value), arterial blood gas analysis was normal and no treatment was given.

The clinical situation progressively worsened over the following 3 yrs. The patient reported dyspnoea (evaluated as grade III New York Heart Association). At this time, DL,CO was $59 \%$ of the predicted value and arterial oxygen tension was $63 \mathrm{mmHg}$ while breathing room air. Diffuse ground-glass opacification was more extensive on HRCT. Because of concern about complications, the patient refused the therapeutic lavage. After being informed about the potential benefits and risks of the treatment the patient agreed to a trial of rituximab therapy. Rituximab was administered in September 2007 as a 1,000 mg intravenous infusion on day 1 and day 15 without any other therapy. Rituximab was well tolerated and induced, as expected, a sharp decrease in the blood B-lymphocyte count which
AFFILIATIONS

* Service de Pneumologie A,

\# Service de Radiologie, Assistance Publique-Hôpitaux de Paris, Hôpital Bichat-Claude Bernard,

+Inserm, Unit 700, Université Paris 7 Faculté de médecine Denis Diderot, Paris, and

'Département d'Immunologie, Centre Hospitalo-universitaire Pontchaillou Rennes, France.

CORRESPONDENCE

R. Borie

Service de Pneumologie A

Hopital Bichat

46 rue Henri Huchard

75877 Paris

Cedex 18

France

Fax: 33140258818

E-mail: raphael.borie@bch.aphp.fr

Received:

October 242008

Accepted after revision:

February 162009

STATEMENT OF INTEREST

None declared.

European Respiratory Journal Print ISSN 0903-1936 Online ISSN 1399-3003 


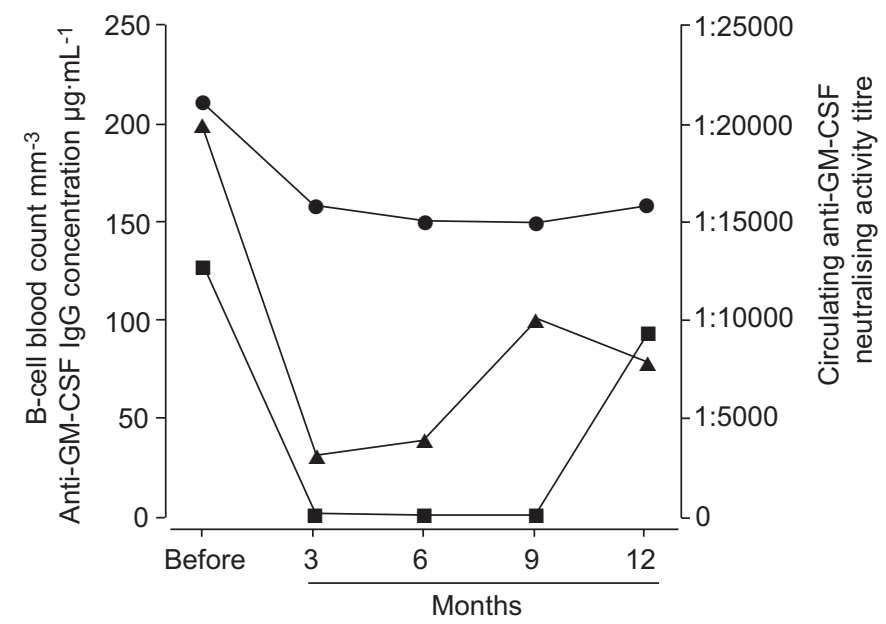

FIGURE 1. Evolution of serum anti-granulocyte-macrophage colony-stimulating factor (anti-GM-CSF) immunoglobulin (Ig)G concentration, and circulating antiGM-CSF neutralising activity titre and B-cell blood counts. The level of anti-GM-CSF activity decreased 3 months after rituximab infusion and reduction was confirmed after 12 months, with a parallel decrease of anti-GM-CSF Ig concentration. After the infusion of rituximab, B-cells were not detectable in the blood for 9 months and reappeared at the last visit of follow-up. $\bullet$ : anti-GM-CSF IgG concentration; $\boldsymbol{\Delta}$ activity; $\mathbf{\square}$ : B-cell blood count.

lasted for 9 months after infusion. At the last follow-up visit $1 \mathrm{yr}$ later, B-lymphocytes were at $96 \mathrm{~mm}^{-3}$. The circulating anti-GM-CSF neutralising activity titre was measured as the serum capacity to neutralise GM-CSF activity on TF1 cells in vitro [2]. Serum anti-GM-CSF IgG concentration and circulating anti-GM-CSF neutralising activity titre decreased 3 months after rituximab infusion (fig. 1). Serum levels of $\gamma$-globulins did not change.

Alveolar-arterial gradient at rest was improved 6 months after rituximab infusion but pulmonary function tests and lung CT images did not improve (table 1). However, 9 months after the initial infusion, the patient reported improved dyspnoea (grade I New York Heart Association). CT images and pulmonary function tests showed a clear improvement that was confirmed on the last follow-up visit 12 months after rituximab infusion. CT images showed a decrease of the diffuse ground-glass opacification in both lower lobes, particularly in the left lower lobe (fig. 2). Lung function tests were also improved with an increase in $\mathrm{DL}, \mathrm{CO}$, alveolar-arterial gradient at rest and desaturation during the 6-min walking test (table 1).

\section{DISCUSSION}

This observation supports the hypothesis that rituximab could be used as an alternative therapy in patients with autoimmune PAP.

The patient's improvement was delayed by 9 months following rituximab infusion, although the decrease in the anti-GMCSF activity was observed 3 month after the infusion. This time difference may be explained by the high titre of circulating anti-GM-CSF neutralising activity observed in this patient and by the delay for recruiting functional macrophages to the alveoli in order to stimulate surfactant clearance. Improvement was clinically, functionally and radiologically evident despite a partial decrease of the circulating anti-GMCSF neutralising activity and serum anti-GM-CSF IgG concentration by ELISA. Available data suggest that the level of GM-CSF autoantibody detected by ELISA does not correlate with the severity of the pulmonary abnormalities at diagnosis [1]. Furthermore there are conflicting results regarding the correlation of anti-GM-CSF concentration and response to treatment with therapeutic lavage or GM-CSF $[5,9]$. To the best of our knowledge, correlation between circulating anti-GMCSF neutralising activity titre and the severity of the disease, or response to therapy, has never been demonstrated. These results support the theory that a partial but sustained reduction of the anti-GM-CSF IgG concentration and circulating anti-GM-CSF neutralising activity could be sufficient to improve alveolar macrophage function and promote resorption of the lipoproteinaceous material in the lung.

Efficacy of rituximab therapy is another step demonstrating that GM-CSF antibodies are pathogenic and mediate PAP development, as already supported by numerous evidences: 1) anti-GMCSF IgG are specifically and strongly associated with PAP; 2) mice deficient in GM-CSF developed a lung disease undistinguishable from human PAP; and 3) purified GM-CSF antibodies reproduced in vitro the myeloid cell abnormalities observed in patients with autoimmune PAP [2, 10, 11]. Interestingly, the clearance of the anti-GM-CSF antibodies through plasmapheresis has been shown to improve PAP in a patient who experienced a failure of the previous treatments with therapeutic lung lavage and GM-CSF therapy [12].

TABLE 1 Lung function testing before and every 3 months after rituximab infusion

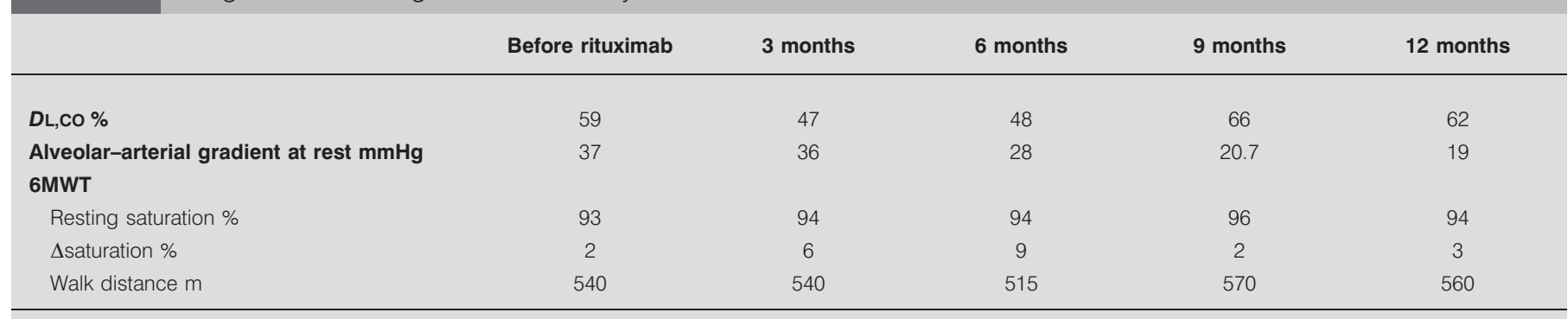

$D \mathrm{~L}, \mathrm{CO}$ : diffusing capacity of the lung for carbon monoxide; $6 \mathrm{MWT}$ : 6-min walking test: $\Delta$ saturation: resting saturation-lowest saturation on $6 \mathrm{MWT}$. 

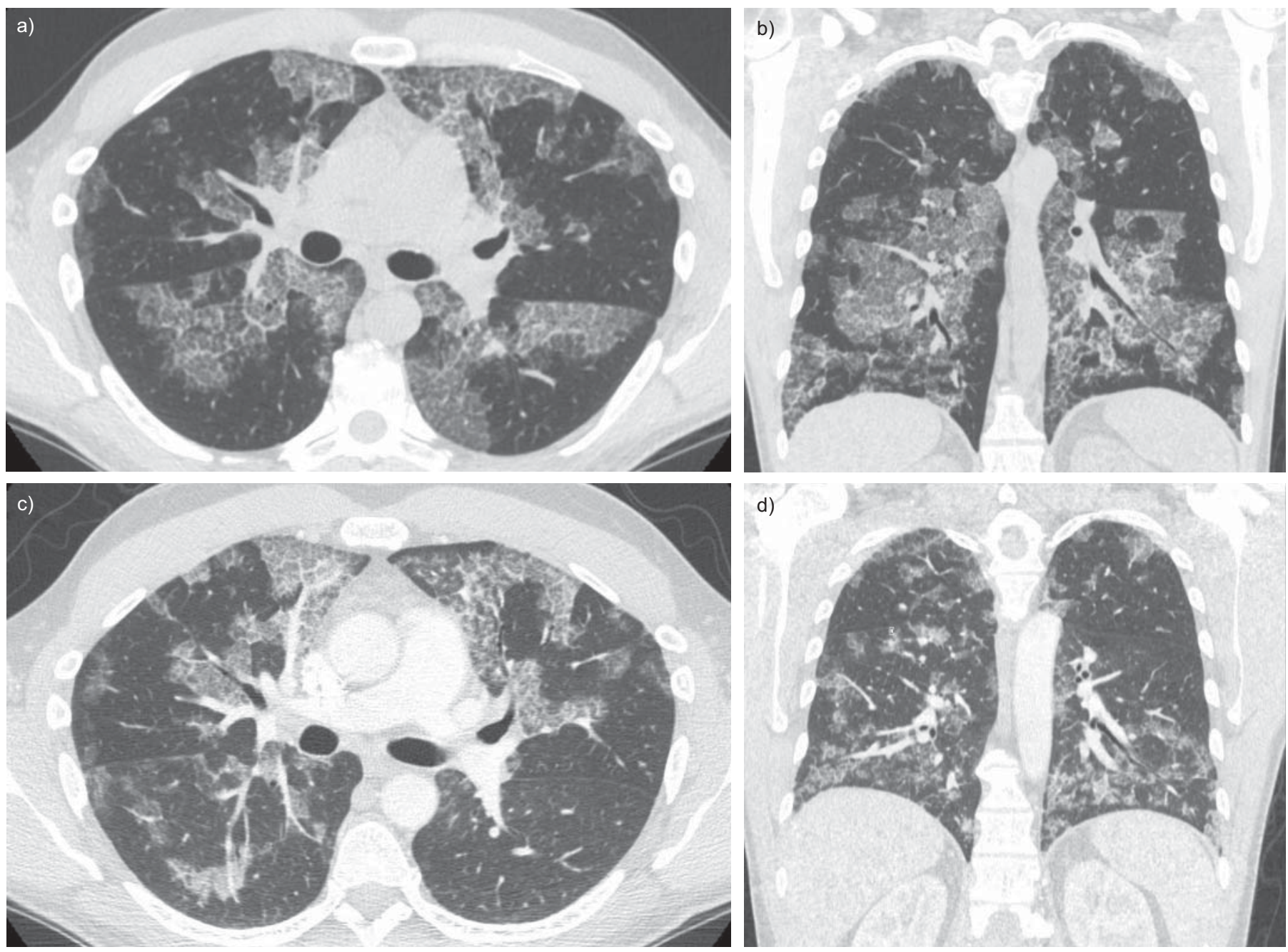

FIGURE 2. High-resolution computed tomography a, b) before and c, d) 9 months after rituximab infusion. Computed tomography images 9 months after rituximab therapy ( $c$ and d) showed a strong decrease of diffuse ground-glass opacification in both lower lobes, particularly in the left lower lobe.

Rituximab has previously demonstrated a good safety profile. In particular, rituximab does not increase the risk of serious infections in rheumatoid arthritis [13], which is a concern in PAP patients who are at risk of secondary bacterial and mycobacterial infections [4].

Finally, as $\sim 28 \%$ of patients with PAP may spontaneously improve [1], we cannot be certain that the improvement observed in this patient was causally related to the beneficial effect of rituximab therapy, although the time-course of improvement supports this conclusion.

This preliminary observation supports the prospective evaluation of the effect of rituximab therapy in autoimmune pulmonary alveolar proteinosis. In view of the delayed improvement observed in this patient, we suggest that this treatment should be evaluated in patients who require non-urgent therapy.

\section{ACKNOWLEDGEMENTS}

B. Crestani designed the protocol; R. Borie, M. Aubier and B. Crestani performed the study; R. Borie and B. Crestani wrote the paper; M-P. Debray reviewed all CT images; and C. Laine performed the anti-GM-CSF dosages.

\section{REFERENCES}

1 Inoue Y, Trapnell BC, Tazawa R, et al. Characteristics of a large cohort of patients with autoimmune pulmonary alveolar proteinosis in Japan. Am J Respir Crit Care Med 2008; 177: 752-762.

2 Uchida K, Beck DC, Yamamoto T, et al. GM-CSF autoantibodies and neutrophil dysfunction in pulmonary alveolar proteinosis. N Engl J Med 2007; 356: 567-579.

3 Trapnell BC, Whitsett JA, Nakata K. Pulmonary alveolar proteinosis. N Engl J Med 2003; 349: 2527-2539.

4 Seymour JF, Presneill JJ. Pulmonary alveolar proteinosis: progress in the first 44 years. Am J Respir Crit Care Med 2002; 166: 215-235.

5 Venkateshiah SB, Yan TD, Bonfield TL, et al. An open-label trial of granulocyte macrophage colony stimulating factor therapy for moderate symptomatic pulmonary alveolar proteinosis. Chest 2006; 130: 227-237.

6 Edwards JC, Szczepanski L, Szechinski J, et al. Efficacy of B-cell-targeted therapy with rituximab in patients with rheumatoid arthritis. N Engl J Med 2004; 350: 2572-2581.

7 Arzoo K, Sadeghi S, Liebman HA. Treatment of refractory antibody mediated autoimmune disorders with an anti-CD20 monoclonal antibody (rituximab). Ann Rheum Dis 2002; 61: 922-924. 
8 Tanaka N, Watanabe J, Kitamura T, et al. Lungs of patients with idiopathic pulmonary alveolar proteinosis express a factor which neutralizes granulocyte-macrophage colony stimulating factor. FEBS Lett 1999; 442: 246-250.

9 Seymour JF, Doyle IR, Nakata K, et al. Relationship of anti-GM-CSF antibody concentration, surfactant protein $\mathrm{A}$ and $\mathrm{B}$ levels, and serum $\mathrm{LDH}$ to pulmonary parameters and response to GM-CSF therapy in patients with idiopathic alveolar proteinosis. Thorax 2003; 58: 252-257.

10 Stanley E, Lieschke GJ, Grail D, et al. Granulocyte/ macrophage colony-stimulating factor-deficient mice show no major perturbation of hematopoiesis but develop a characteristic pulmonary pathology. Proc Natl Acad Sci USA 1994; 91: 5592-5596.

11 Kitamura T, Uchida K, Tanaka N, et al. Serological diagnosis of idiopathic pulmonary alveolar proteinosis. Am J Respir Crit Care Med 2000; 162: 658-662.

12 Kavuru MS, Bonfield TL, Thomassen MJ. Plasmapheresis, GM-CSF, and alveolar proteinosis. Am J Respir Crit Care Med 2003; 167: 1036.

13 Doran MF, Crowson CS, Pond GR, et al. Predictors of infection in rheumatoid arthritis. Arthritis and Rheum 2002; 46: 2294-2300. 